\title{
BMJ Open Validation of the Kinyarwanda-version Short-Form Leeds Dyspepsia Questionnaire and Short-Form Nepean Dyspepsia Index to assess dyspepsia prevalence and quality-of-life impact in Rwanda
}

\author{
Arcade Nkurunziza, ${ }^{1}$ Vincent Dusabejambo, ${ }^{1,2}$ Kelly Everhart, ${ }^{3}$ Steve Bensen, ${ }^{3,4}$ \\ Tim Walker ${ }^{1,5}$
}

To cite: Nkurunziza $A$, Dusabejambo V, Everhart K, et al. Validation of the Kinyarwanda-version ShortForm Leeds Dyspepsia Questionnaire and ShortForm Nepean Dyspepsia Index to assess dyspepsia prevalence and quality-of-life impact in Rwanda. BMJ Open 2016;6:e011018. doi:10.1136/bmjopen-2015011018

- Prepublication history and additional material is available. To view please visit the journal (http://dx.doi.org/ 10.1136/bmjopen-2015011018).

Received 5 January 2016 Revised 26 April 2016 Accepted 16 May 2016

CrossMark

For numbered affiliations see end of article.

Correspondence to Dr Tim Walker; timwalkerd@gmail.com

\section{ABSTRACT}

Objectives: We aimed to develop and validate Kinyarwanda versions of Short-Form Leeds Dyspepsia Questionnaire (SF-LDQ) and Short-Form Nepean Dyspepsia Index (SF-NDI) to measure the frequency and severity of dyspepsia and associated quality-of-life impact in Rwanda.

Setting: A single, tertiary care centre in Rwanda.

Participants: 200 consecutive Kinyarwanda-speaking patients referred to endoscopy (100 patients) or medical outpatients (100 patients).

Interventions: Kinyarwanda versions of the SF-LDQ and SF-NDI were developed from English versions by translation, with back translation, crosschecking and pilot testing. Study participants completed these questionnaires at enrolment (time 1), and then completed the surveys again with blinded phone interviewers 3 days later (time 2). 20 randomly selected participants, diagnosed with a peptic ulcer on index endoscopy, completed a third survey by phone at day 30 (time 3), after therapy.

Primary outcome measures: Internal consistency at time 1 (by Cronbach's $\alpha$ ) and test-retest reliability between time 1 and time 2 (Spearman's correlation coefficient) for translated SF-LDQ and SF-NDI; validity versus clinical diagnosis (by receiver operating characteristic (ROC) curve) and responsiveness to treatment for SF-LDQ (by change in mean score). All outcomes were measured as per protocol.

Results: Cronbach's $\alpha$ of the translated SF-LDQ was 0.93 , showing high internal consistency. Spearman's correlation coefficient comparing time 1 and time 2 was $0.978(p<0.001)$, demonstrating high reliability. Cronbach's $\alpha$ for the translated SF-NDI was 0.92. A cut-off score of 16 on the SF-LDQ showed a sensitivity of $97 \%$ and a specificity of $71 \%$ for the diagnosis of dyspepsia, correctly classifying $89 \%$ of patients. In the responsiveness analysis, the mean SF-LDQ score was reduced from 20.1 prior to treatment to 13.9 after 30 days of treatment $(p=0.003)$.

\section{Strengths and limitations of this study}

- Both dyspepsia symptom severity and quality-of-life impact measured concurrently in the same patient population.

- Study staff were blinded to time 1 survey results when administering time 2 surveys.

- $100 \%$ participant follow-up achieved from time 1 to time 2.

- No gold standard comparison available to validate Short-Form Nepean Dyspepsia Index (SF-NDI), the dyspepsia quality-of-life tool, meaning that surrogate markers had to be used.

- Survey administration methods differed between time 1 and time 2: interpersonal interviews and phone-based interviews.

Conclusions: The Kinyarwanda versions of the SFLDQ and SF-NDI were valid, reliable and responsive to treatment.

\section{INTRODUCTION}

Dyspepsia is a constellation of upper gastrointestinal symptoms that present a significant personal, social and financial burden to patients and healthcare resources worldwide. ${ }^{12}$ Although no standard, universal definition of dyspepsia adequately characterises the symptom complex across diverse cultural and sociodemographic environments; clinicians tend to rely on the presence of chronic, recurrent epigastric pain or discomfort as a platform for the diagnosis and management of patients presenting to primary and subspecialty healthcare with upper gastrointestinal symptoms. ${ }^{3}$ 
The differential diagnosis of non-specific upper gastrointestinal symptoms is broad. In the absence of alarm features, patients with chronic, recurrent upper abdominal pain or discomfort who have yet to undergo additional clinical evaluation are identified with a preliminary diagnosis of uninvestigated dyspepsia. In a meta-analysis of cross-sectional surveys reporting the prevalence of uninvestigated dyspepsia, Ford et at report a global prevalence of $20.8 \%$ (n 312415 ; range 1.8$57 \%$; $95 \%$ CI $17.8 \%$ to $23.9 \%$ ), identifying significantly increased prevalence with a broad definition of dyspepsia, female gender, use of tobacco or non-steroidal antiinflammatory drugs (NSAIDs), and confirmed infection with Helicobacter pylori.

Esophagogastroduodenoscopy (EGD) remains the gold standard approach to the investigation of dyspepsia. Patients with evidence of structural disease on EGD are considered to have organic dyspepsia; gastrooesophageal reflux disease (GERD) and peptic ulcer disease (PUD) are among the most common endoscopic diagnoses associated with dyspeptic symptoms worldwide. ${ }^{5-10}$ Patients with dyspepsia without evidence of structural disease despite thorough clinical evaluation are diagnosed with functional dyspepsia (FD); the chronic symptom complex of FD is likely multifactorial, and is often attributed to a combination of visceral hypersensitivity and upper gastrointestinal dysmotility that varies with each individual and with time. ${ }^{11}$ Unlike dyspepsia of organic origin, FD is not associated with an increased risk of mortality; however, it is significantly associated with decrements in health-related quality of life (HR-QoL). ${ }^{12}$

\section{Rwanda}

Despite growing evidence of significant disease burden, notably including gastric malignancy, $H$. pylori infection and PUD, associated with dyspeptic symptoms in Rwanda $^{13}$ and elsewhere in sub-Saharan Africa, ${ }^{8}$ there remains a paucity of population-based data characterising the epidemiology and clinical course of organic and FD in these locations. Ford $e t a l^{14}$ calculated the prevalence of dyspepsia in excess of $35 \%$ (n 1421; 95\% CI $19.2 \%$ to $54 \%$ ) from two surveys ${ }^{15}{ }^{16}$ administered in Nigeria; if these data are representative of the African continent, the prevalence of uninvestigated dyspepsia in Africa approaches double that of the global population.

Although EGD is the tool of choice to investigate dyspepsia in Rwanda, there are few facilities and trained providers equipped to provide EGD to Rwandan patients in this resource-limited healthcare setting. No other clinical tools are currently available to Rwandan healthcare workers to adequately assess symptom severity, symptom frequency or HR-QoL of patients with dyspepsia, limiting the diagnosis, management and investigation of dyspepsia in a culturally competent manner. However, as Rwanda is a small, centralised country that uses a single traditional language (Kinyarwanda) in addition to English, it is ideally suited for the use of a patient- completed questionnaire as a surrogate or adjunct to EGD in primary and subspecialty healthcare settings.

\section{Tool selection}

The Leeds Dyspepsia Questionnaire and the Nepean Dyspepsia Index, and their short-form equivalents (SF-LDQ and SF-NDI), are self-reported item-based questionnaires that were developed in English to quantify dyspeptic symptom severity and frequency and HR-QoL related to FD, respectively. ${ }^{17}{ }^{18}$ Specifically, the SF-LDQ captures the frequency and severity of upper abdominal discomfort, heartburn, regurgitation and nausea over the preceding 2 months. Each item is assigned a numerical score that is summed into a total score; scores $>14$ have been indicative of dyspepsia in other populations. The SF-NDI evaluates tension/ anxiety, interference with daily activities, disruption of usual eating/drinking, knowledge of/control over disease symptoms and interference with work/study with two-item five-point Likert scales, with a total score calculated as the mean of the five subscale scores. ${ }^{19}$

Owing to their simplicity and brevity, there is robust precedent for translation and validation of the SF-LDQ and SF-NDI for use in non-English-speaking populations. ${ }^{20}$ Notably, Mahadeva and colleagues translated and validated the SF-LDQ ${ }^{21}$ and SF-NDI ${ }^{22}$ into Malay and Malaysian English for use in a multiethnic Asian population with dyspepsia, and reported adequate reliability (internal consistency determined by Cronbach's $\alpha$ : 0.8 and 0.74 ; test-retest reliability determined by Spearman's coefficient: 0.98), validity (area under the receiver operating characteristic (ROC) curve: 0.71 and 0.77 ) and responsiveness to treatment (mean LDQ score reduced following treatment with proton pump inhibitor (PPI): 17.0-14.0, p 0.08 in Malay; 18.0-11.0, p 0.008 in Malaysian English) for both versions of the translated LDQ questionnaire (n 310). For translated versions of the SF-NDI, Mahadeva et $a t^{2}$ reported adequate internal consistency and test-retest reliability (Cronbach's $\alpha$ : $0.83-0.90$; Spearman's coefficient: 0.83 and 0.90 ), and approximate validity with correlation to the 36-Item Short-Form Health Survey (SF-36), a validated, widely used clinical tool that measures generic HR-QoL (n 143).

\section{Study objectives}

The objective of this study was to develop and validate a reliable translation of the SF-LDQ and SF-NDI in Kinyarwanda for use in epidemiological and clinical applications in primary and subspecialty healthcare settings in Rwanda. This study also assessed the responsiveness of the Kinyarwanda version of the SF-LDQ to treatment in patients diagnosed with dyspepsia.

\section{METHODS}

We used a prior Malaysian study validating dyspepsia tools $^{21} 22$ as a model for the translation, prospective cross-sectional survey administration and psychometric 
evaluation of the SF-LDQ and SF-NDI into Kinyarwanda. The study protocol was reviewed and approved by the Kigali University Teaching Hospital (KUTH) Ethics Committee.

\section{Instrument translation}

We selected three medically experienced colleagues who were fluent in both languages to translate the tools from English into Kinyarwanda. The study author, supervisor and English-Kinyarwanda translators met to analyse the language and content of the English tools to guide culturally appropriate translation. Once consensus was achieved, the translated tools were back-translated from Kinyarwanda to English by a separate team of three qualified translators in order to verify that the Kinyarwanda version of the tools maintained the integrity of the English versions. The study author, supervisor and translators again met to discuss the language and content of the back-translated tools. The corrected Kinyarwanda versions of both tools were then evaluated by two independent Kinyarwanda linguistic experts, who made corrections to the translated tools. The final Kinyarwanda versions of the SF-NDI and SF-LDQ were then completed by $10 \mathrm{KUTH}$ employees ( 5 nurses, 3 administrators, 2 service personnel) selected to represent diverse age (mean age 35 years; range $18-52$ years) and sociodemographic backgrounds (7 female; 7 with university-level education) in a pilot test of the translated tool. None of the participants in the pilot administration of either tool encountered any difficulties with the Kinyarwanda translation, completing both tools without assistance. Therefore, no further changes were made to either tool prior to their use with study participants.

\section{Instrument administration}

We recruited adult patients (age $>17$ years; $\mathrm{n} 200$ ) who presented to outpatient medical care at KUTH, a national referral hospital in Rwanda (n 100) or who awaited EGD at the same location (n 100). The study author and a trained research assistant approached patients in the waiting area of outpatient clinics and the endoscopic suite, explaining the purpose of the research and asking if they would like to be considered for enrolment in the study. Patients were excluded from the study if they reported a history of abdominal surgery, major medical disease requiring tertiary medical care or current major psychiatric disease. Patients were also excluded from the study if they did not adequately speak and understand Kinyarwanda. Enrolled participants underwent a process of informed consent, agreeing to fill out the study tools at that time (time 1) and to be contacted by phone by study administrators to complete the tool a second time (time 2). Participants who were literate in Kinyarwanda were given a printed copy of the questionnaires to complete themselves at time 1. Trained personnel orally administered the tools to participants who were not literate in Kinyarwanda at time 1, and to all participants over the phone at time
2. Study personnel were blinded to time 1 survey results until all time 2 data were collected.

\section{Data collection}

Time 1 data were collected at the time of participant enrolment at KUTH between November 2014 and January 2015. Each participant completed a Kinyarwanda version of the SF-LDQ and SF-NDI, as well as basic demographic information. Time 2 data were collected 3 days later; participants were contacted by telephone by the study author or a trained research assistant and asked to orally complete a Kinyarwanda version of the SF-LDQ and SF-NDI.

In order to test the responsiveness of the SF-LDQ to treatment, we randomly selected 20 patients who underwent EGD and were diagnosed with PUD. These patients completed the SF-LDQ a third time (time 3), following 1 month of oral PPI therapy with or without additional triple therapy for $H$. pylori infection.

\section{Data analysis}

The translated Kinyarwanda dyspepsia questionnaires were evaluated by assessing their reliability. Additionally, the validity and responsiveness of the SF-LDQ were assessed. We used SPSS V.16.0 and Excel to compute the statistical parameters reported in this study. Participants who did not complete the time 1 and time 2 surveys in full were excluded from analysis.

Specifically, the internal consistency and test-retest reliability of the translated SF-LDQ and SF-NDI were determined by calculating Cronbach's $\alpha$ for time 1 and time 2 scores and Spearman's correlation coefficient between time 1 and time 2 scores, respectively. The validity of the SF-LDQ was determined against the gold standard of clinical diagnosis using ROC curves. There is no gold standard for the measurement of HR-QoL; therefore, the validity of the translated SF-NDI was estimated first against SF-LDQ scores collected at time 1 during this study using ROC curves, and then using the Mann-Whitney U test to establish known groups' construct validity of the total and five subscale scores of the SF-NDI relative to the severity (increased symptom severity defined as $\mathrm{SF}-\mathrm{LDQ} \geq 15$ ) of dyspeptic symptoms. ${ }^{22} \mathrm{We}$ assessed the responsiveness of the SF-LDQ using Wilcoxon rank matched-pair testing by comparing the time 1 and post-treatment scores of 20 patients who were diagnosed with PUD on EGD, underwent 30 days of PPI therapy and completed the tool a third time.

\section{RESULTS}

The final Kinyarwanda-translated versions of the SF-LDQ and SF-NDI tools are presented as online supplementary appendices 1 and 2.

A total of 200 study participants were enrolled between November 2014 and January 2015. The mean age of enrolled patients was 41 years. A majority of patients in the overall cohort were diagnosed with 
dyspepsia by a clinician (true dyspepsia prevalence among study participants 69\%), including all of the patients awaiting EGD. Most patients were residents of Kigali $(61 \%)$ and $62 \%$ were female (see table 1 ).

\section{Short-Form Leeds Dyspepsia Questionnaire}

The response rates for the SF-LDQ and SF-NDI at time 1 and time 2 were $100 \%$. Cronbach's $\alpha$ was calculated at time 1 and time 2 to assess the internal consistency of the translated SF-LDQ, revealing a value of 0.93 at time 1 and 0.92 at time 2. The Spearman's correlation coefficient between time 1 and time 2 scores on the SF-LDQ was 0.978. Response frequencies for each item on the SF-LDQ are shown in table 2.

The summed total score of the SF-LDQ at time 1 was compared to the gold standard of clinical diagnosis by the treating physician, using an ROC curve (figure 1). The point along the ROC curve that correctly classified most participants was chosen as the SF-LDQ cut-off score for the diagnosis of dyspepsia. This SF-LDQ cut-off score of 16 showed a sensitivity of $97 \%$ and a specificity of $71 \%$ for the diagnosis of dyspepsia, correctly classifying $89 \%$ of study participants ( $\kappa$ coefficient 0.75 ).

\begin{tabular}{lc}
\hline Table 1 Demographic characteristics of study population \\
\hline Characteristic & Number (\%) \\
\hline Dyspepsia & $137(68)$ \\
No dyspepsia & $63(32)$ \\
Gender & \\
Female & $123(62)$ \\
Male & $77(38)$ \\
Residency & \\
Kigali & $121(60)$ \\
East & $28(14)$ \\
West & $8(4)$ \\
South & $18(9)$ \\
North & $25(13)$ \\
Education & \\
None & $18(9)$ \\
Primary & $70(35)$ \\
Secondary & $63(32)$ \\
University & $49(25)$ \\
Occupation & \\
Jobless & $37(19)$ \\
Farmer & $48(24)$ \\
Student & $26(13)$ \\
Private & $46(26)$ \\
Public & $34(17)$ \\
Retired & $9(4)$ \\
Marital status & \\
Single & $69(34)$ \\
Married & $105(53)$ \\
Widowed & $15(7)$ \\
Divorced & $6(3)$ \\
Separated & $5(3)$ \\
Having children & $138(69)$ \\
Children & $62(31)$ \\
No children & \\
\hline Residency refers to province of residence. \\
\end{tabular}

Among the 20 patients with PUD who received PPI therapy and were again interviewed at time 3 , the mean SF-LDQ score changed from 20.1 prior to treatment (time 1) to 13.9 after 1 month of therapy (time 3), with a $p$ value of 0.003 by Wilcoxon rank matched-pair testing.

\section{Short-Form Nepean Dyspepsia Index}

Cronbach's $\alpha$ for the SF-NDI was 0.96 at time 1 and 0.95 at time 2. The Spearman's correlation coefficient between time 1 and time 2 scores on the SF-NDI was 0.89 .

The validity of the SF-NDI was first estimated by comparison of the per-patient total scores on the SF-NDI and SF-LDQ using ROC curves plotted against clinical diagnosis (figure 2). The area under each curve was similar (0.91 for SF-LDQ vs 0.89 for SF-NDI), and no statistical difference was apparent between the two curves $(\mathrm{p}=0.35)$.

Known groups' construct validity of the total and subscale scores of the Kinyarwanda version of the SF-NDI was established relative to the severity of dyspeptic symptoms using the Mann-Whitney $\mathrm{U}$ test. For all five subscale scores and the total score of the SF-NDI, there was significant $(\mathrm{p}<0.001)$ compromise of HR-QoL for patients with severe relative to patients with mild dyspeptic symptoms (table 3 ).

\section{DISCUSSION}

This study demonstrates that tools developed for the study of dyspepsia prevalence and its impact on HR-QoL in Western populations can be successfully adapted for use in an African language and cultural context. Obtained results indicate that Kinyarwanda versions of the SF-LDQ and SF-NDI are reliable and internally consistent and that the SF-LDQ displays a high correlation with African physicians' clinical diagnoses, with $89 \%$ of patients correctly classified by an SF-LDQ $>16$ (area under the ROC curve 0.91 ). While objective proof of the quality-of-life impact measured by the SF-NDI was more difficult to obtain, secondary markers suggest a high correlation between SF-NDI and SF-LDQ scores, as well as high internal consistency and reliability for the SF-NDI. Finally, the SF-LDQ was responsive to changes with treatment in patients likely to respond to acid suppression, with a clinically and statistically significant fall in both scores in patients with clinically diagnosed PUD following initiation of a PPI.

The strengths of this study lie in clear and rigorous validation methodology applied to a sub-Saharan linguistic and cultural context with significant dyspepsia-associated disease burden but without clinical precedent for evaluative tools available to treating physicians. This study's administration of both tools in written, oral and phone-based forms realistically reflects the modes of communication that are routinely and necessarily employed for clinical and research purposes 
Table 2 Time 1 response frequencies for the SF-LDQ

\begin{tabular}{lll}
\hline & Frequency (\%) & Severity (\%) \\
\hline Indigestion & & $44(22)$ \\
Not at all & $38(19)$ & $14(7)$ \\
Less than once a month & $14(7)$ & $21(10.5)$ \\
Between once a month and once a week & $23(11.5)$ & $44(22)$ \\
Between once a week and once a day & $42(21)$ & $77(38.5)$ \\
Once a day and more & $83(41.5)$ & $74(37)$ \\
Heartburn & & $19(9.5)$ \\
Not at all & $65(32.5)$ & $25(12.5)$ \\
Less than once a month & $18(9)$ & $34(17)$ \\
Between once a month and once a week & $28(14)$ & $48(24)$ \\
Between once a week and once a day & $37(18.5)$ & $80(40)$ \\
Once a day or more & $52(26)$ & $25(12.5)$ \\
Regurgitation & & $27(13.5)$ \\
Not at all & $80(40)$ & $36(18)$ \\
Less than once a month & $22(11)$ & $32(16)$ \\
Between once a month and once a week & $25(12.5)$ & $73(36.5)$ \\
Between once a week and once a day & $38(19)$ & $20(20)$ \\
Once a day and more & $35(17.5)$ & $24(12)$ \\
Nausea & & $35(17.5)$ \\
Not at all & $69(34.5)$ & $48(24)$ \\
Less than once a month & $15(7.5)$ & \\
Between once a month and once a week & $24(12)$ & $40(20)$ \\
Between once a week and once a day & $52(26)$ & \\
Once a day or more & &
\end{tabular}

in Rwanda, ensuring that clinicians can confidently employ these tools without concern for compromised results. Both tools were chosen for their simplicity and ease of use, further reducing survey length and complexity, barriers which can otherwise prove insurmountable in real-world African settings, where clinical demands often compete with research for the limited healthcare worker resources available. Additionally, the simultaneous evaluation of dyspeptic symptom prevalence and

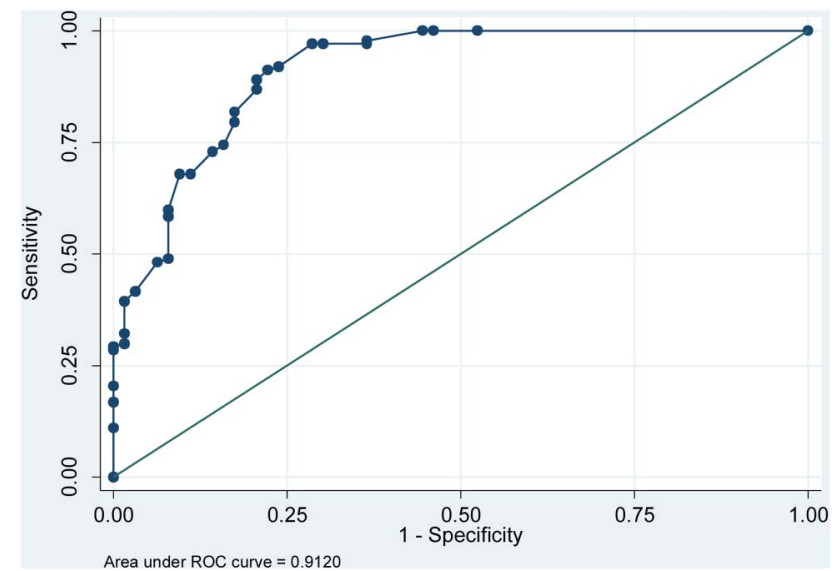

Figure 1 ROC curve for SF-LDQ total score at time 1 against clinical diagnosis. ROC, receiver operating characteristic; SF-LDQ, Short-Form Leeds Dyspepsia Questionnaire.
HR-QoL enables this study to demonstrate for the first time that these domains are closely correlated in an African population, a link that bears important clinical and healthcare policy implications as Rwanda adapts to treat this patient population.

Although this is the first validation of the SF-LDQ and SF-NDI in Africa, similar studies have been performed in Malaysia and China; ${ }^{21}{ }^{23}$ together with the initial validation studies of these tools in Western populations; ${ }^{17-1924}$

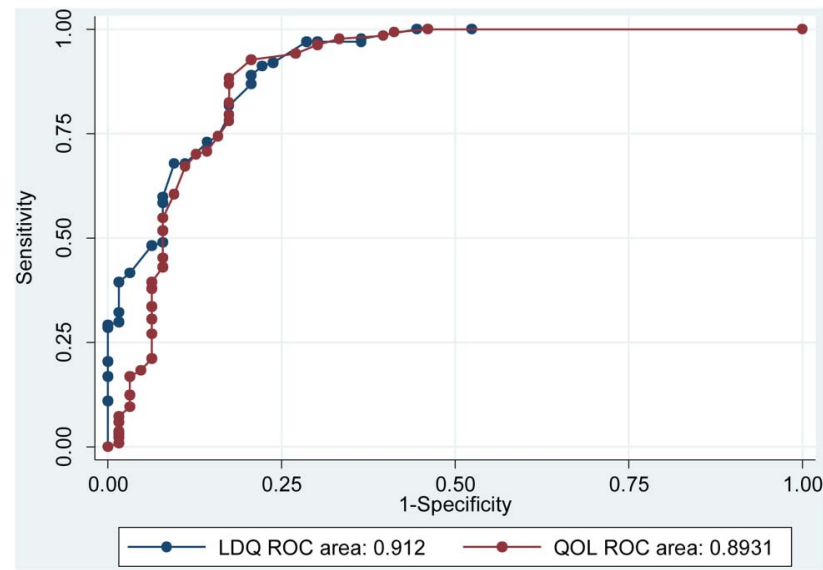

Figure 2 ROC curves for SF-NDI and SF-LDQ at time 1 against clinical diagnosis. ROC, receiver operating characteristic; SF-LDQ, Short-Form Leeds Dyspepsia Questionnaire; SF-NDI, Short-Form Nepean Dyspepsia Index; QoL, quality of life. 
Table 3 Known groups' construct validity of the Kinyarwanda version of the SF-NDI relative to the severity of dyspeptic symptoms*

\begin{tabular}{|c|c|c|c|}
\hline SF-NDI subscale scores & $\begin{array}{l}\text { Mild } \\
\text { (n 14t) }\end{array}$ & $\begin{array}{l}\text { Severe } \\
\text { (n 152) }\end{array}$ & p Value \\
\hline Tension (median; range) & $2(2-6)$ & $5(2-10)$ & $<0.001$ \\
\hline Interference (median; range) & $2(2-4)$ & $6(2-10)$ & $<0.001$ \\
\hline Eating/drinking (median; range) & $3(2-5)$ & $6(2-10)$ & $<0.001$ \\
\hline Knowledge/control (median; range) & $2(2-4)$ & $3(2-10)$ & 0.001 \\
\hline Work/study (median; range) & $2(2-6)$ & $6(2-10)$ & $<0.001$ \\
\hline Total (median; range) & $25(20-44)$ & $56(20-92)$ & $<0.001$ \\
\hline
\end{tabular}

these global results serve as a benchmark for the use of long-form and short-form versions of the LDQ and NDI in multiple languages and varied populations.

Specifically, LDQ translations to Malay, Malaysian English and Mandarin ${ }^{21} 23$ performed similar to the current study in terms of reliability (Spearman's coefficient $0.78-0.98$ ), with a range of internal consistency (Cronbach $\alpha$ 0.74-0.80) lower than results reported for this study population. Critically, LDQ results in historical studies of primary and secondary care populations were less valid (when compared with clinical diagnoses) than those obtained in this study (area under the ROC curves ranging from 0.71 to 0.84 ), save for a single Italian version of the SF-NDI (Cronbach's $\alpha$ 0.90, Pearson's correlation coefficient 0.92 , sensitivity $80 \%$ and specificity $82 \%) .{ }^{25}$

Therefore, within the context of these geographically and demographically comparable validation studies, the results of this initiative to develop tools to measure the prevalence of dyspepsia and its impact on HR-QoL in sub-Saharan Africa impress with their robust validity. This relative success may be attributed to a number of observations, including differences in patient presentation, as African patients tend to present later in the course of other diseases, ${ }^{26}$ differences in patient population, as this study enrolled patients at a tertiary care centre, or differences in the cultural expression of dyspeptic symptoms. ${ }^{27}$ It is also possible that the tools developed by this study are more culturally intelligible than those deployed in prior research settings, given the meticulous, multidisciplinary methods by which they were translated.

All research initiatives are subject to limitations. In this study, no gold standard for dyspepsia-related quality of life has been developed in Kinyarwanda; therefore, the validity of the SF-NDI was evaluated with surrogate SF-LDQ scores and contemporaneous clinical diagnoses. As this study focused exclusively on patients seeking medical care at a tertiary healthcare centre, it is possible that the Kinyarwanda version of the SF-LDQ might prove less discriminatory in other populations; however, the wide range of SF-LDQ and SF-NDI scores and the significant prevalence of incidental dyspepsia in the medical outpatient population (which likely resembles 'primary' dyspepsia) suggest a diversity of patient illness experience that is reassuring. Finally, the initial administration of these tools (verbal or written) depended on the literacy of each enrolled patient; all patients completed the surveys by telephone on readministration. Although this heterogeneity could potentially have reduced the testretest reliability of these tools, in fact reliability remained encouragingly high in our final study analysis. Further investigation of dyspepsia in African populations, with attendant translations of these tools into other African languages, will prove instructive areas for future research.

\section{CONCLUSION}

The Kinyarwanda versions of the SF-LDQ and SF-NDI developed by this study proved reliable and valid, particularly when compared to the gold standard of clinical diagnosis. These tools are recommended for use in clinical and research initiatives involving the prevalence of dyspepsia and its impact on HR-QoL in Kinyarwandaspeaking patients of sub-Saharan Africa.

Author affiliations

${ }^{1}$ College of Medicine and Health Sciences, University of Rwanda, Huye, Rwanda

${ }^{2}$ Kigali University Teaching Hospital, Kigali, Rwanda

${ }^{3}$ Geisel School of Medicine, Dartmouth College, Hanover, New Hampshire, USA

${ }^{4}$ Dartmouth-Hitchcock Medical Center, Lebanon, New Hampshire, USA

${ }^{5}$ Butare University Teaching Hospital, Huye, Rwanda

Acknowledgements The authors thank Professor Nick Talley and Professor Brendan Delaney for kind permission to translate the SF-NDI and SF-LDQ tools.

Contributors TW conceived the study. AN, VD and TW designed the study. AN and VD carried out and supervised the interviews. AN, VD, KE, SB and TW interpreted the data. AN, KE, SB and TW drafted the manuscript. AN, VD, $K E, S B$ and TW critically revised the manuscript for intellectual content. All authors read and approved the final manuscript. TW and AN are guarantors of the paper.

Funding This study was partially funded by the Rwandan Ministry of Health, via a Masters of Medicine (Internal Medicine) Research Grant. The views expressed are those of the authors and not of the Rwandan Ministry of Health. 
Competing interests None declared.

Patient consent Obtained.

Ethics approval The study was approved by the Kigali University Teaching Hospital Ethics Committee.

Provenance and peer review Not commissioned; externally peer reviewed.

Data sharing statement A database containing deidentified patient-level data for all study participants' response to administered questionnaires at all time points is available from the corresponding author upon request.

Open Access This is an Open Access article distributed in accordance with the Creative Commons Attribution Non Commercial (CC BY-NC 4.0) license, which permits others to distribute, remix, adapt, build upon this work noncommercially, and license their derivative works on different terms, provided the original work is properly cited and the use is non-commercial. See: http:// creativecommons.org/licenses/by-nc/4.0/

\section{REFERENCES}

1. Brook RA, Kleinman NL, Choung RS, et al. Functional dyspepsia impacts absenteeism and direct and indirect costs.

Clin Gastroenterol Hepatol 2010;8:498-503.

2. Lacy BE, Weiser KT, Kennedy AT, et al. Functional dyspepsia: the economic impact to patients. Aliment Pharmacol Ther 2013;38:170-7.

3. Ford AC, Bercik P, Morgan DG, et al. The Rome III criteria for the diagnosis of functional dyspepsia in secondary care are not superior to previous definitions. Gastroenterol 2014;146:932-40.

4. Ford AC, Marwaha A, Sood R, et al. Global prevalence of, and risk factors for, uninvestigated dyspepsia: a meta-analysis. Gut 2015 Jul;64:1049-57.

5. Mahadeva S, Goh KL. Clinically significant endoscopic findings in a multi-ethnic population with uninvestigated dyspepsia. Dig Dis Sci 2012;57:3205-12.

6. Samaila A, Okeke E, Malu A. Endoscopic findings and clinical predictors of organic disease among patients with dyspepsia in Jos, Nigeria. Nig J Gastroenterol Hepatol 2011;3:39-46.

7. Faintuch JJ, Silva FM, Navarro-Rodriguez T, et al. Endoscopic findings in uninvestigated dyspepsia. BMC Gastroenterol 2014;14:19.

8. Ayana SM, Swai B, Maro VP, et al. Upper gastrointestinal endoscopic findings and prevalence of Helicobacter pylori infection among adult patients with dyspepsia in northern Tanzania. Tanzan $J$ Health Res 2014;16:16-22.

9. Vasiliou C, Xiromeritou V, Kafiri G, et al. Endoscopic and histological findings and Helicobacter pylori status in patients with reflux and/or dyspeptic symptoms: a recent Greek cohort study. Gastroenterol Nurs 2014;37:431-8.

10. Afihene MK, Denyer M, Amuasi JH, et al. Prevalence of Helicobacter pylori and Endoscopic findings among Dyspeptics in Kumasi, Ghana. Open Sci J Clin Med 2014;2:63.
11. Lee KJ, Kindt S, Tack J. Pathophysiology of functional dyspepsia. Best Pract Res Clin Gastroenterol 2004;18:707-16.

12. Aro P, Talley NJ, Agréus L, et al. Functional dyspepsia impairs quality of life in the adult population. Aliment Pharmacol Ther 2011;33:1215-24.

13. Walker TD, Karemera M, Ngabonziza F, et al. Helicobacter pylori status and associated gastroscopic diagnoses in a tertiary hospital endoscopy population in Rwanda. Trans R Soc Trop Med Hyg 2014 May;108:305-7.

14. Ford AC, Talley NJ. Epidemiology of dyspepsia. In: Talley NJ, Locke GR, Moayyedi P, West JJ, Ford AC, Saito YA, eds. Gl epidemiology: diseases and clinical methodology. 2nd edn. Chichester: WileyBlackwell, 2014:158-71.

15. Holcombe C, Omotara BA, Padonu MK, et al. The prevalence of symptoms of dyspepsia in north eastern Nigeria. A random community based survey. Trop Geogr Med 1991;43:209-14.

16. Ihezue $\mathrm{CH}$, Oluwole FS, Onuminya JE, et al. Dyspepsias among the highlanders of Nigeria: an epidemiological survey. Afr J Med Med Sci 1996;25:23-9.

17. Moayyedi P, Duffett S, Braunholtz D, et al. The Leeds Dyspepsia Questionnaire: a valid tool for measuring the presence and severity of dyspepsia. Aliment Pharmacol Ther 1998;12:1257-62.

18. Talley NJ, Haque M, Wyeth JW, et al. Development of a new dyspepsia impact scale: the Nepean Dyspepsia Index. Aliment Pharmacol Ther 1999;13:225-36.

19. Talley NJ, Verlinden M, Jones M. Quality of life in functional dyspepsia: responsiveness of the Nepean Dyspepsia Index and development of a new 10-item short form. Aliment Pharmacol Ther 2001;15:207-16.

20. Anastasiou F, Antonakis N, Chaireti G, et al. Identifying dyspepsia in the Greek population: translation and validation of a questionnaire. BMC Public Health 2006;6:56

21. Mahadeva S, Chan WK, Mohazmi M, et al. Validation study of the Leeds Dyspepsia Questionnaire in a multi-ethnic Asian population J Gastroenterol Hepatol 2011;26:1669-76.

22. Mahadeva S, Wee HL, Goh KL, et al. Quality of life in South East Asian patients who consult for dyspepsia: validation of the short form Nepean Dyspepsia Index. Health Qual Life Outcomes 2009;7:45.

23. Leow HR, Ching SM, Sujarita R, et al. Mandarin version of the Leeds Dyspepsia Questionnaire: a valid instrument for assessing symptoms in Asians. J Dig Dis 2014;15:591-6.

24. Fraser A, Delaney BC, Ford AC, et al. The short-form Leeds Dyspepsia Questionnaire validation study. Aliment Pharmacol Ther 2007;25:477-86.

25. Gatta L, Moayyedi P, Tosetti C, et al. A validation study of the Italian Short-Form Leeds Dyspepsia Questionnaire. Intern Emerg Med 2010;5:501-6.

26. Kigozi IM, Dobkin LM, Martin JN, et al. Late-disease stage at presentation to an HIV clinic in the era of free antiretroviral therapy in Sub-Saharan Africa. J Acquir Immune Defic Syndr 2009;52:280-9.

27. Yap P, Mahadeva S, Goh KL. The influence of cultural habits on the changing pattern of functional dyspepsia. Dig Dis 2014;32:217-21. 